\title{
The molecular mechanisms of Aloin induce gastric cancer cells apoptosis by targeting High Mobility Group Box I
}

\author{
Hong Tao ${ }^{1,2}$ \\ Tuo Tang ${ }^{1,2}$ \\ Shengnan Wang ${ }^{1,2}$ \\ Ziqian Wang ${ }^{1,2}$ \\ Yunfei $\mathrm{Ma}^{\mathrm{l}, 2}$ \\ Tianyu Cai ${ }^{1,2}$ \\ Xiuliang Cheng ${ }^{1,2}$ \\ Shimei $Q i^{1,2}$ \\ Yao Zhang ${ }^{1,2}$ \\ Zhilin $\mathrm{Qi}^{1,2}$ \\ 'Department of Biochemistry and \\ Molecular Biology, Wannan Medical \\ College, Wuhu, Anhui 241002, People's \\ Republic of China; ${ }^{2}$ Anhui Province Key \\ Laboratory of Active Biological \\ Macro-Molecules, Wannan Medical \\ College, Wuhu, Anhui 241002, People's \\ Republic of China
}

This article was published in the following Dove Press journal: Drug Design, Development and Therapy

Purpose: Aloin (ALO), a bioactive ingredient extracted from aloe vera, has anti-tumor effects. High Mobility Group Box 1 (HMGB1), a highly conserved nuclear DNA-binding protein, has been implicated in various cancer types. Highly expressed HMGB1 is closely associated with tumor cells apoptosis, proliferation and migration. We investigated the specific molecular mechanisms by which ALO-induced apoptosis by targeting HMGB1 in gastric cancer cells.

Materials and methods: Human gastric cancer HGC-27 cells were treated with different doses of ALO $(100,200$ and $400 \mu \mathrm{g} / \mathrm{ml})$ for $24 \mathrm{~h}$, after which DAPI staining was used to observe the nuclear morphology, Annexin V/PI double staining assay was used to determine the rate of apoptosis; Western blotting was used to detect the levels of PARP, pro-caspase3, HMGB1 and RAGE; nuclear translocation of HMGB1 was determined by conducting a nucleoplasm separation experiment. The Enzyme linked immunosorbent assay (ELISA) assay was used to detect release of HMGB1. The HGC-27 cells, transfected with HMGB1 shRNA plasmids, were stimulated with ALO for $24 \mathrm{~h}$, after which a flow cytometry assay was used to detect the rate of apoptosis. HGC-27 cells were pre-treated with or without ALO and then stimulated with rhHMGB1, the phosphorylation of Akt, mTOR, P70S6K, S6, 4EBP1, ERK, P90RSK, cAMP regulatory element binding (CREB) were detected by Western blotting.

Results: After different doses of ALO treatment, the nuclei showed morphological changes characteristic of apoptosis. Apoptotic rates were enhanced in a dose dependent manner. The level of cleaved PARP was enhanced and pro-caspase3, HMGB1 and RAGE levels were reduced, HMGB1 nuclear translocation and release were inhibited. The activation of rhHMGB1-induced Akt-mTOR-P70S6K and ERK-CREB signalling pathways was inhibited by ALO. Blocking these signalling pathways by special inhibitors and HMGB1 knockdown could enhance ALO-induced HGC-27 cell apoptosis.

Conclusion: ALO- induced HGC-27 cell apoptosis by down-regulating expressions of HMGB1 and RAGE, inhibiting HMGB1 release and then suppressing rhHMGB1-induced activation of Akt-mTOR-P70S6K and ERK-P90RSK-CREB signalling pathways.

Keywords: Aloin, gastric cancer, HMGB1, Akt, mTOR, P70S6K, ERK, P90RSK, CREB, apoptosis

\section{Introduction}

Department of Biochemistry and Molecular Biology, Wannan Medical College, No. 22 Wenchang West Road, Wuhu, Anhui 241002, People's Republic of China

Email zhangyao@ahedu.gov.cn;

422627721@qq.com
Gastric cancer is a common malignancy tumor in China, and is associated with high morbidity and mortality. At present, chemotherapy, radiotherapy and surgery are the main treatments for gastric cancer. However, due to the lack of early diagnostic markers and the characteristics of easy metastasis, the therapeutic effects against 
gastric cancer is remain unsatisfactory. ${ }^{1,2}$ Therefore, searching for biological targets for early diagnosis of gastric cancer will assist gastric cancer treatment and prognosis.

The High Mobility Group Box 1 (HMGB1) is a highly conserved nuclear DNA-binding protein. ${ }^{3}$ As a nuclear protein, HMGB1 plays an important role in DNA repair, transcription and differentiation. ${ }^{4}$ HMGB1 can also be released into the extracellular space by active secretion or passive release. ${ }^{5,6}$ As an extracellular signal molecule, HMGB1 induces "secondary biological effects" by binding with its receptors, such as RAGE or TLRs, after which it is involved in the onset and development of various diseases, including inflammation and cancer. ${ }^{7,8}$ HMGB1 is highly expressed in many malignant tumors and reportedly plays an important role in tumor apoptosis, proliferation and metastasis. It is also considered an early biological target of many malignant tumors. ${ }^{9,10}$ Inhibition of HMGB1 enhances hepatoma cell apoptosis induced by doxorubicin. ${ }^{11}$ In addition, the correlation of HMGB1 and gastric cancer has been reported. In gastric cancer, highly expressed HMGB1 promotes the cell proliferation and migration by activating NFкВ and ERK signal pathways, ${ }^{12,13}$ HMGB1 silencing sensitises cells to oxaliplatin and induces MGC-803 cell apoptosis. ${ }^{8}$ These studies suggest that HMGB1 may be considered a novel therapeutic target.

Aloin (ALO), is a natural compound extracted from aloe vera. It has anti-cancer abilities and can inhibit cell proliferation and induce apoptosis of lung, colorectal and breast cancers. ${ }^{14-16}$ Our previous study also showed that ALO could induce gastric cancer cell apoptosis by activating the MAPKs signalling pathway. ${ }^{17}$ However, it is unknown whether ALO induces apoptosis of gastric cancer cells by targeting HMGB1.

In this study, we mainly explored the role of HMGB1 in ALO-induced gastric cancer cells apoptosis and further investigated the underlying molecular mechanism. Our data shows that ALO induces apoptosis in gastric cancer HGC-27 cells via decreasing the expression and release of HMGB1, inhibiting the activation of rhHMGB1-induced Akt-mTOR-P70S6K and ERK-P90RSK-cAMP regulatory element binding (CREB) signalling pathways. This study will provide experimental basis for HMGB1 as a therapeutic target for gastric cancer and provide a new perspective for the effect of ALO on apoptosis of gastric cancer cells.

\section{Materials and methods}

\section{Antibodies and reagents}

ALO, Rapamycin, LY294002, U0126 all were purchased from Selleck Chemicals (Houston,TX, USA), rhHMGB1 was purchased from Sigma-Aldrich (Merck KGaA, Darmstadt, Germany), DAPI was obtained from Thermo Fisher Scientific, Inc. (Waltham, MA, USA). Human HMGB1 ELISA kit was bought from CUSABIO (Wuhan, China); CCK-8 kit and Annexin V/PI apoptotic kit were purchased from KeyGen Biotech Co., Ltd. (Nanjing, China); Primary antibodies of $\beta$-actin, PARP, pro-caspase3, cleaved caspase3, p-Akt, Akt p-mTOR, mTOR, p-P70S6K, P70S6K, p-S6, S6, p-4EBP1, 4EBP1, p-ERK, p-P90RSK, p-CREB all were purchased from Cell Signalling Technology (Beverly, MA, USA), Secondary antibodies coupled to IRDye800 fluorophore for use with the Odyssey Infrared Imaging System were purchased from LI-COR Biosciences (Lincoln, Nebraska, USA). Image $\mathrm{J}$ version 1.52 software (National Institutes of Health) was used for the densitometry analysis.

\section{Cell culture}

A human undifferentiated gastric cancer HGC-27 cell line was bought from GuangZhou Cellcook Biotech Co., Ltd. (Guangzhou, China). The cells were cultured in RPMI-1640 medium (Gibco; Thermo Fisher Scientific, Inc., Waltham, MA, USA) containing $10 \%$ fetal bovine serum (FBS; Lonsera, South America), 10\% non-essential amino acids (NEAA), $100 \mu \mathrm{g} / \mathrm{ml}$ streptomycin and $100 \mathrm{U} / \mathrm{ml}$ penicillin (Beyotime Institute of Biotechnology, Haimen, China), and incubated in a cell incubator at $37^{\circ} \mathrm{C}$ and $5 \% \mathrm{CO}_{2}$.

\section{Cell counting kit-8 (CCK-8) assay}

Cell viability was detected by CCK- 8 kit according to the manufacturer's protocol. In brief, HGC-27 cells were planted in 96 well cell culture plates, following incubation with different concentrations of $\operatorname{ALO}(25,50,100,200$, $300,400 \mu \mathrm{g} / \mathrm{ml}$ ) for $24 \mathrm{~h}$. We added $10 \mu \mathrm{l} /$ well of CCK-8 working fluid, and after incubation for another $2 \mathrm{~h}$, the absorbance at $450 \mathrm{~nm}$ was measured using a Multiskan ${ }^{\mathrm{TM}}$ GO plate reader (Thermo Fisher Scientific, Inc.). These data are shown as mean $\pm \mathrm{SD}$.

\section{Morphological examination of apoptotic nuclei using DAPI staining}

HGC-27 cells were seeded in 12 well cell culture plates, after treatment with different doses of $\operatorname{ALO}(100,200$, 
$400 \mu \mathrm{g} / \mathrm{ml}$ ) for $24 \mathrm{~h}$, the cell medium was discarded. The cells in the 12 well plates were washed with PBS and fixed with $4 \%$ paraformaldehyde for $30 \mathrm{~min}$ at room temperature followed by DAPI staining in the dark. The nuclei morphology was observed and photographed under inverted fluorescence microscope (Olympus, Japan).

\section{Annexin V-FITC/PI double staining assay}

Apoptotic rate was determined by Annexin V/PI double staining assay. Briefly, after treatment, HGC-27 cells were collected and twice washed with PBS. Cells were resuspended in $500 \mu \mathrm{l}$ of binding buffer, then $5 \mu \mathrm{PI}$ and $5 \mu \mathrm{l}$ Annexin V -fluorescein isothiocyanate (FITC) were added. After staining in the dark, apoptotic rate was detected by flow cytometry (BD Biosciences, Franklin Lakes, NJ, USA).

\section{Nucleoplasm separation experiment}

After treatment with ALO, the cytoplasmic and nuclear proteins of HGC-27 cells were extracted using a Nuclear and Cytoplasmic Protein Extraction kit (P0028; Beyotime Institute of Biotechnology) according to the instruction provided by the manufacturer.

\section{Western blotting}

After treatment, HGC-27 cells were rinsed with cold PBS three times and then lysed in RIPA buffer with added protease inhibitors (Beyotime Institute of Biotechnology, Haimen, China) for $30 \mathrm{~min}$ on ice. Lysates were centrifuged $(12,000 \mathrm{rpm})$ at $4^{\circ} \mathrm{C}$ for $15 \mathrm{~min}$, after which the supernatant was collected. Protein concentration was determined by bicinchoninic acid assay. Equal total protein of each sample was loaded onto 10 or $12 \%$ gels for SDS-PAGE and transferred to nitrocellulose membranes (Pall Corporation, Port Washington, NY, USA), which were blocked with 5\% skim milk powder in TBST for $1 \mathrm{~h}$ at room temperature, then incubated with the indicated primary antibodies overnight at $4^{\circ} \mathrm{C}$. After washing three times in TBST, the membranes were incubated with IRDye800 fluorophore-conjugated secondary antibody for $1 \mathrm{~h}$ at room temperature in the dark. The protein expressions were detected using the LI-COR Odyssey Infrared Imaging System (LI-COR Biosciences) and Image $\mathrm{J} 1.52$ software was used to quantify the proteins.

\section{Enzyme linked immunosorbent assay (ELISA)}

HGC-27 cells were incubated with ALO for $24 \mathrm{~h}$. After this, the cell medium was collected, and HMGB1 levels in cell culture supernatant were examined using a human HMGB1 ELISA kit (CUSABIO, Wuhan, China), as per the instructions of the manufacture. Each treatment was performed in triplicate, the resultant data are shown as mean $\pm \mathrm{SD}$.

\section{HMGBI interference plasmids and cell transfection}

HMGB1 interference plasmids and negative plasmids were purchased from GeneChem Co., Ltd. (Shanghai, China). HGC-27 cells were seeded in 6 well cell culture plates. When the cell density reached $80 \%$, HMGB1 interference and negative plasmids were transfected using the Lipofectamine ${ }^{\circledR} 3000$ Reagent (Thermo Fisher Scientific, Inc.). Operations were performed according to the manufacturer's instructions. The interference efficiency was detected by using Western blotting.

\section{Statistical analysis}

All data are expressed as mean \pm SD. Statistical analyses were performed using SPSS Version 17.0 software (SPSS, Inc., Chicago, IL, USA). The results were compared using one-way ANOVA. $P<0.05$ was taken as a statistically significant difference.

\section{Results}

\section{Aloin reduced HGC-27 cells viability and induced cellular apoptosis or death}

We first detected the effect of ALO on HGC-27 cell viability. HGC-27 cells viability was clearly inhibited after ALO treatment, and the inhibitory effect was concentration dependent (Figure 1A). According to this result, we selected 100, 200 and $400 \mu \mathrm{g} / \mathrm{ml}$ of ALO for the subsequent experiments. Secondly, the effects of ALO on HGC-27 cell apoptosis were detected through DAPI staining and Annexin V/PI double staining assays. These results showed that the nuclear sizes were uniform, and the cells of the control group had a light blue, round nuclear morphology. However, ALO treated cells showed different nuclear sizes and clear nuclear condensation (Figure 1B). Flow cytometry assay data showed that the apoptotic rate was $2.83 \%$ in the control group, whereas it was 7.73 after $100 \mu \mathrm{g} / \mathrm{ml}$ ALO, 14.49 after $200 \mu \mathrm{g} / \mathrm{ml} \mathrm{ALO}$ and $23.04 \% 400 \mu \mathrm{g} / \mathrm{ml}$ ALO treatments (Figure 1C). Additionally, ALO-induced expression of cleaved PARP increased, while pro-caspase3 levels of decreased in a dose dependent manner (Figure 1D). Taken together, these results suggested that ALO could induce apoptosis of gastric cancer HGC-27 cells. 

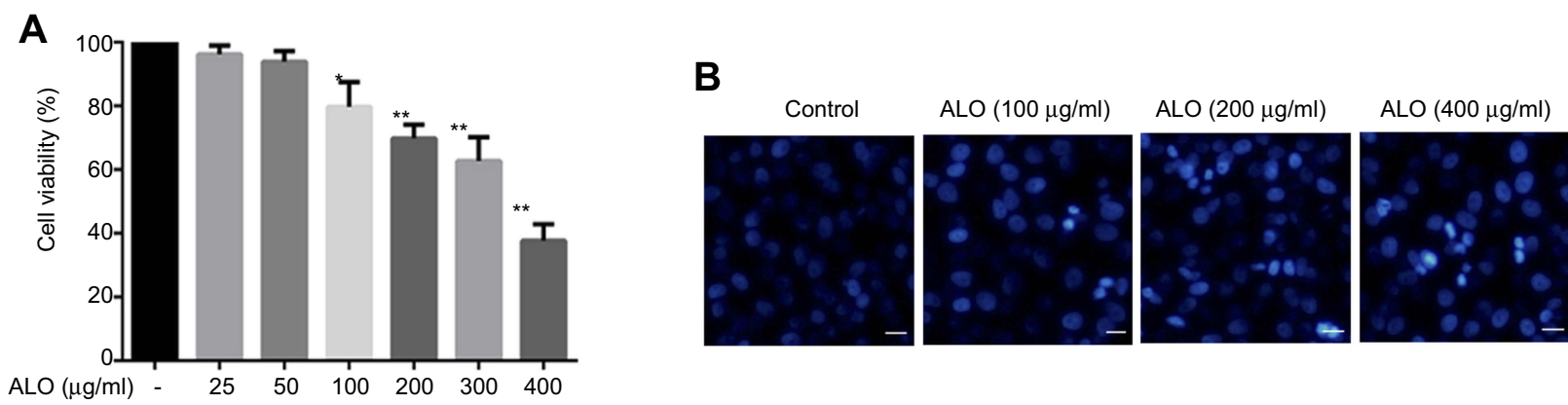

C
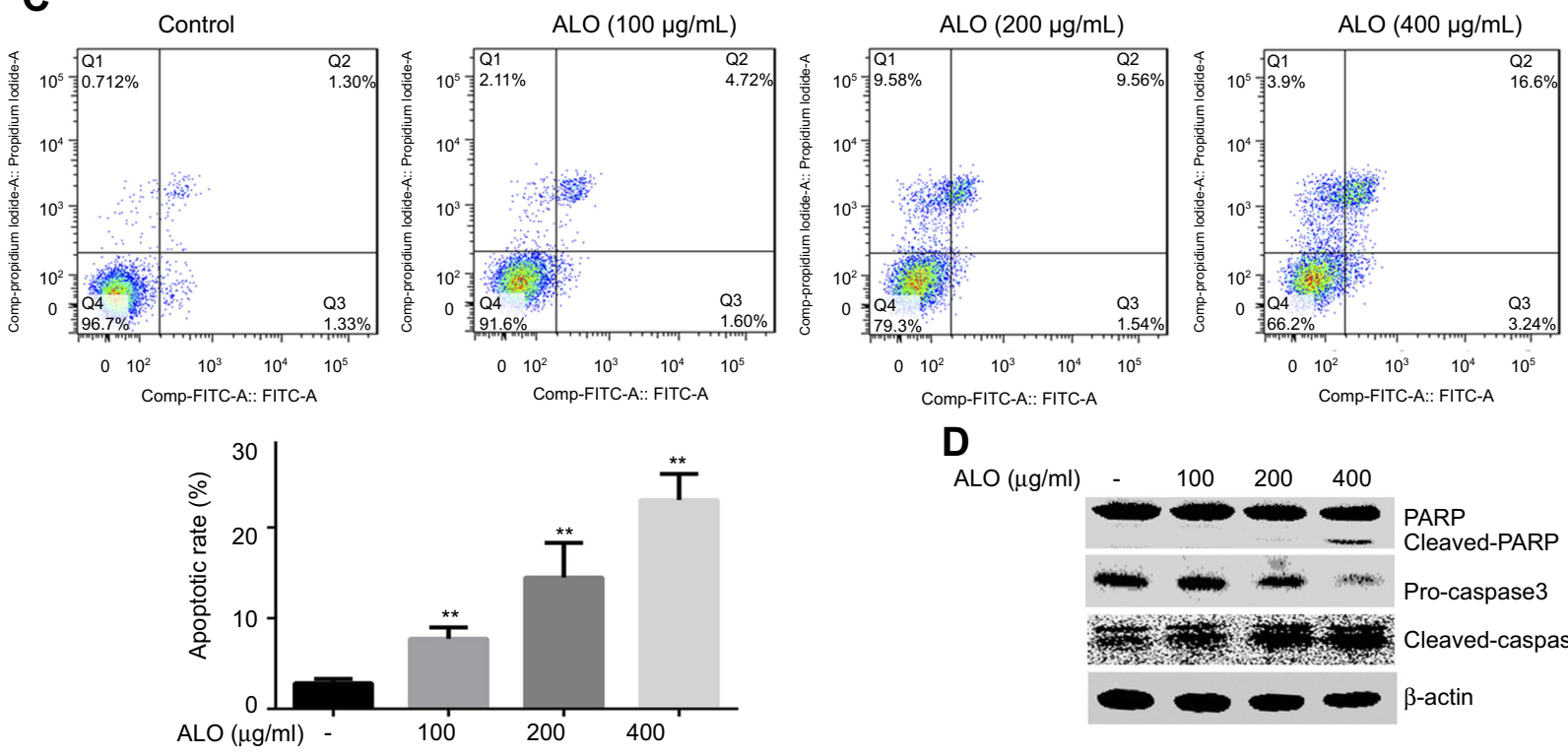

D

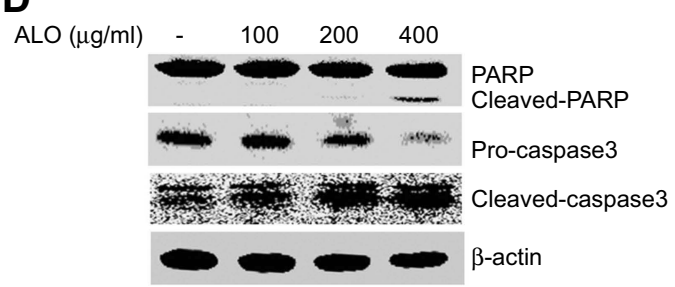

Figure I ALO reduced HGC-27 cell viability and induced cell apoptosis or death. HGC-27 cells were treated with different doses of ALO for 24 h, after which the cell viability was determined by CCK-8 assay (A). Nuclear morphology was observed using DAPI staining, magnification $\times 100$; Scale bar, I00 $\mu \mathrm{m}(\mathbf{B})$. The apoptotic rate of HGC27 cells was detected using a flow cytometry assay. The experiment was independently repeated for three times, and the representative results are shown (C). The expressions of PARP and pro-caspase 3 were determined by Western blotting, $\boldsymbol{\beta}$-actin was used as a loading control (D). Data are presented as mean \pm SD of three independent experiments. ${ }^{*} p<0.05$ and $* * p<0.01$ vs the control group.

Abbreviation: ALO, aloin.

Aloin reduced the expression and release of HMGBI in HGC-27 cells

During tumor development, HMGB1 acts as both a pro- and anti-tumor protein by either promoting or suppressing tumor growth, proliferation, angiogenesis, invasion and metastasis. ${ }^{18}$ In order to prove the correlation between HMGB1 and gastric cancer, we detected the total level of HMGB1 in normal gastric epithelial cells GES-1 and gastric cancer cells HGC27 using Western blotting. Our results showed that HMGB1 expression in HGC-27 cells was higher than that in GES-1 cells (Figure 2A). This result implied that HMGB1 might act as a target for gastric cancer treatment.

We further examined the effect of ALO on HMGB1 expression and release. The levels of total HMGB1 and RAGE were detected by Western blotting, the release of HMGB1 was determined by using ELISA assay, and the nuclear translocation of HMGB1 was examined usig a nucleoplasm separation experiment. Figure 2B shows that ALO reduced the expression of HMGB1 in a dose dependent manner. In addition, our results also indicated that the HMGB1 level in cell culture supernatant was decreased after ALO treatment (Figure 2C). In the control group, the cytoplasmic HMGB1 level was higher than that in the ALO group, while the expression of HMGB1 in nuclear proteins showed the opposite change (Figure 2D). Collectively, these results suggested that ALO not only inhibited HMGB1 expression, but also blocked HMGB1 release.

\section{Down-regulation of HMGBI expression enhanced Aloin induced HGC-27 cells}

\section{apoptosis}

It has been reported that knockdown HMGB1 by shRNA transfection or inhibition of HMGB1 release enhanced 
A

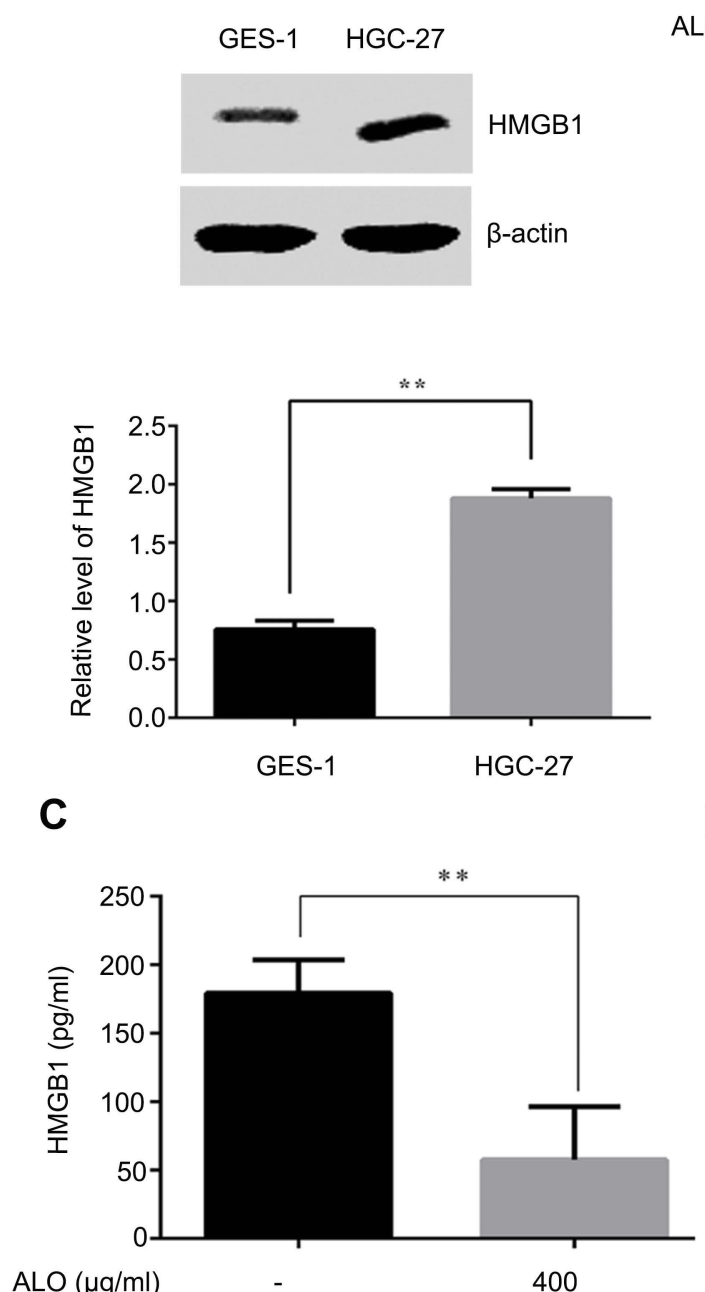

B
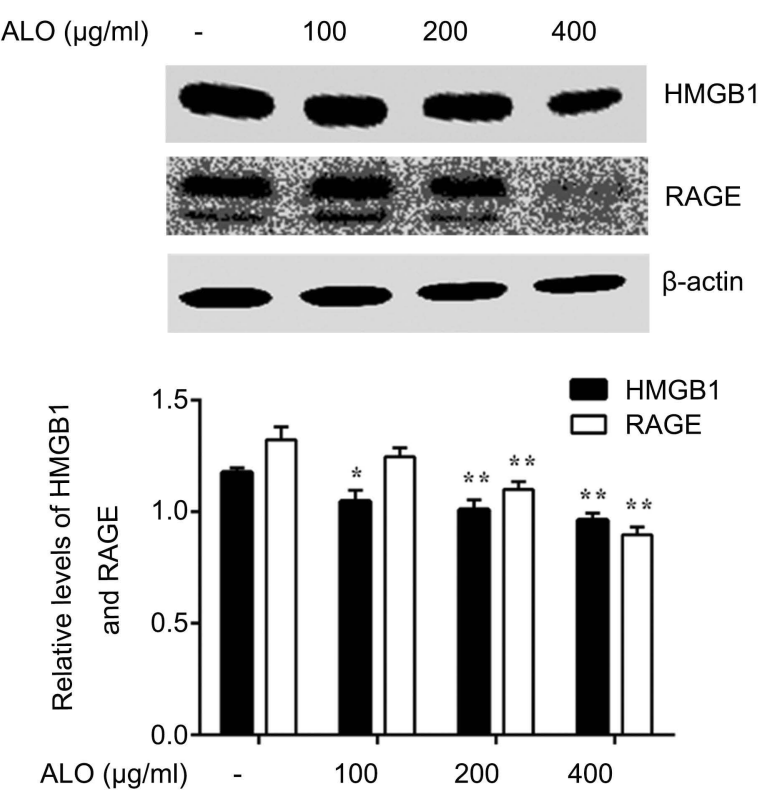

D
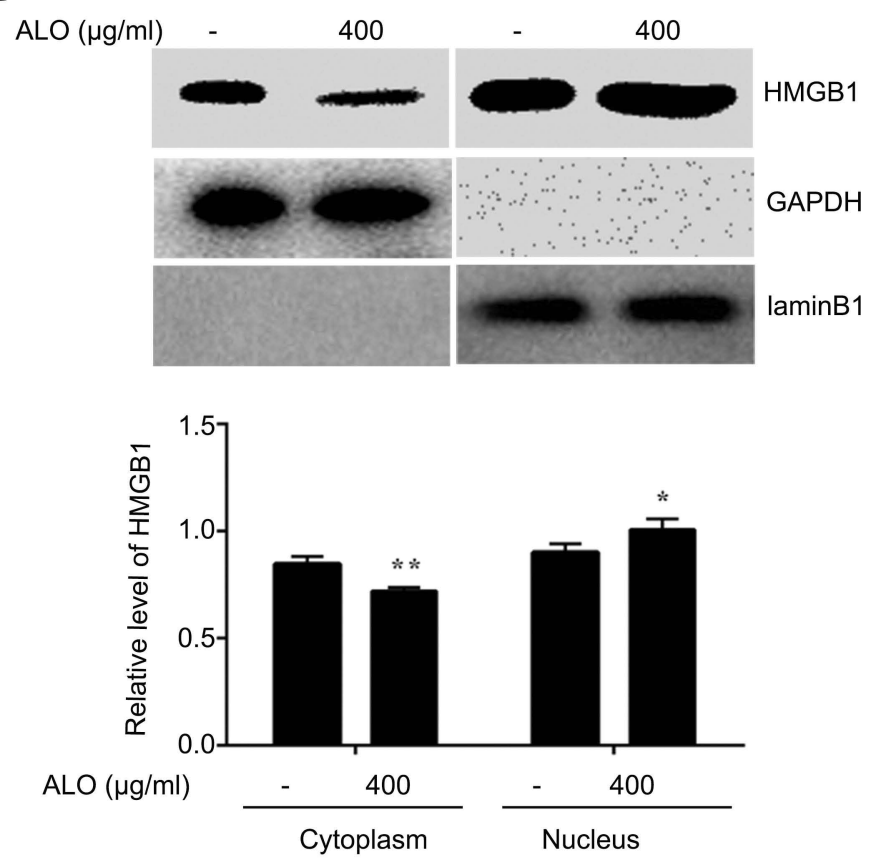

Figure 2 ALO suppressed the expression and release of HMGBI in HGC-27 cells. HMGBI levels in normal gastric epithelial GES-I and gastric cancer HGC-27 cells were detected by Western blotting (A). Data are presented as mean \pm SD of three independent experiments. ** $p<0.01$ vs GES-I cells. HGC-27 cells were treated with different doses of ALO (100, 200 and $400 \mu \mathrm{g} / \mathrm{ml})$ for $24 \mathrm{~h}$, total proteins and cell culture supernatant were extracted and the expressions of HMGBI and RAGE were determined by using Western blotting (B). HMGBI release was determined by ELISA assay (C). Nuclear translocation of HMGBI was detected by completion of a nucleoplasm separation experiment (D). Data are presented as mean \pm SD of three independent experiments. ${ }^{*} p<0.05$ and $* * p<0.01$ vs control group.

Abbreviation: ALO, aloin.

melphalan-induced the apoptosis of human pancreatic cancer Panc2.03 cells and colon cancer HCT116 cells. ${ }^{19}$ In gastric cancer HGC-27 cells, we sought to determine whether HMGB1 affected ALO-induced apoptosis. HGC-27 cells were transfected with HMGB1 shRNA and negative plasmids respectively. After $48 \mathrm{~h}$ transfection, the cells were stimulated with $400 \mu \mathrm{g} / \mathrm{ml}$ of ALO for $24 \mathrm{~h}$. The apoptotic rate was detected by flow cytometry assay. As shown in Figure 3A, in cells transfected with negative plasmids, the apoptotic rate was $44.58 \%$ after ALO treatment. However, the rate of apoptosis increased to $59.86 \%$ in HMGB1 shRNA transfected cells. The interference efficiency of HMGB1 shRNA is shown in Figure 
A
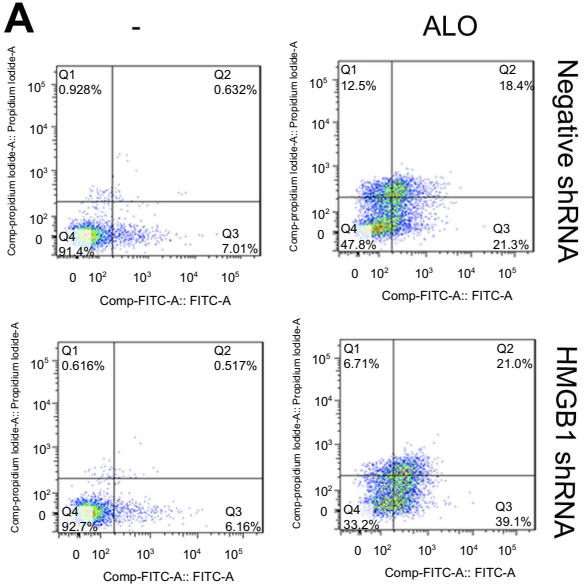

B

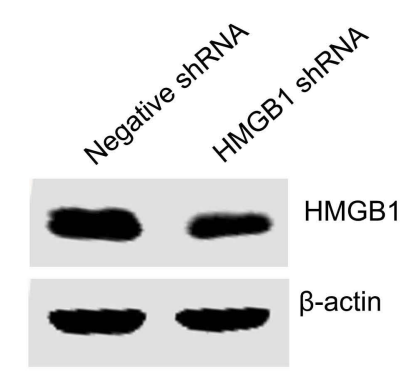

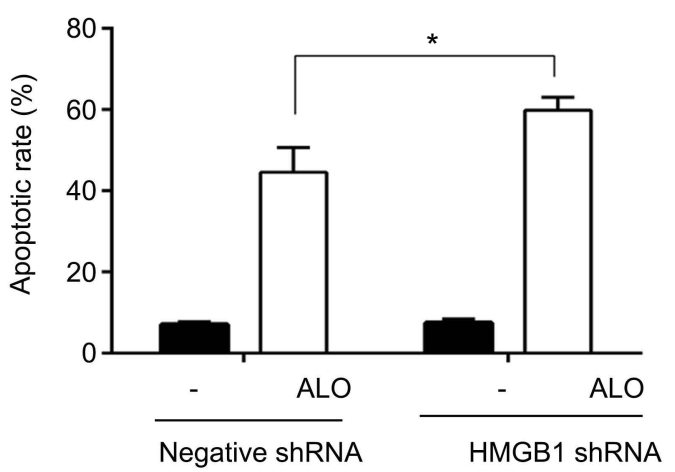

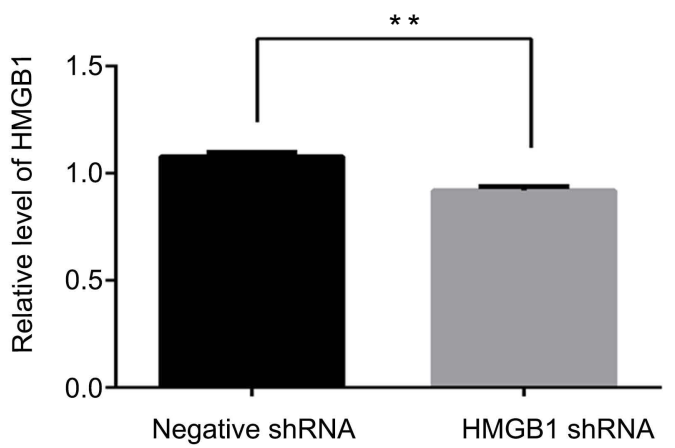

Figure 3 Down-regulation of HMGBI enhanced ALO-induced HGC-27 cell apoptosis. HGC-27 cells were transfected with negative and HMGBI shRNA plasmids respectively. After $48 \mathrm{~h}$ transfection, the cells were treated with $400 \mu \mathrm{g} / \mathrm{ml}$ of ALO for $24 \mathrm{~h}$, after which the apoptotic rates were detected by flow cytometry (A). After transfection, total proteins were extracted, and HMGBI interference efficiency was examined by Western blotting (B). The experiment was repeated three times and data are shown in mean $\pm S D$. $* p<0.05$ and $* * p<0.01$ vs the negative plasmids transfection group.

Abbreviation: ALO, aloin.

3B. This result signified that down-regulation of HMGB1 enhanced ALO-induced HGC-27 apoptosis.

\section{Aloin inhibited rhHMGBI-induced activation of Akt-mTOR-P70S6K and ERK-P90RSK-CREB signalling pathways}

Our above results showed that ALO could reduce the expression levels of HMGB1 and its receptor RAGE and inhibit the release of HMGB1. Additionally, HMGB1 acts as an extracellular signal molecule can induce many signal pathways including MAPKs, Akt and NF-KB activation through binding with receptors. ${ }^{8,20}$ Among the important signal molecules, Akt and ERK have an important relationship with cancer initiation and progression. ${ }^{21}$ Thus, we further investigated whether ALO affected HMGB1-induced Akt and ERK signalling pathways activation. HGC-27 cells were first treated with different doses of rhHMGB1 $(0.5,1$ and $2 \mu \mathrm{g} / \mathrm{ml})$ for $12 \mathrm{~h}$, the phosphorylation of Akt and ERK signaling pathways were detected by Western blotting. Figures $4 \mathrm{~A}$ and $5 \mathrm{~A}$ show that rhHMGB1 enhanced the phosphorylation of AktmTOR-P70RSK and ERK-P90RSK-CREB in a dose dependent manner. Secondly, HGC-27 cells were treated with $2 \mu \mathrm{g} / \mathrm{ml}$ of rhHMGB1 for the different times $(3,6,12$ and $24 \mathrm{~h}$ ), the activation of these signal pathways was also detected by using Western blot. As shown in Figure 4B, the phosphorylation of Akt, mTOR and P70S6K began to increase around $3 \mathrm{~h}$, peaking at $6 \sim 12 \mathrm{~h}$ and then decreased around $24 \mathrm{~h}$. The activation of ERK, P90RSK and CREB also showed the same change (Figure 5B). Lastly, HGC-27 cells were pre-treated with different doses of ALO for $2 \mathrm{~h}$, and then stimulated with rhHMGB1 for $12 \mathrm{~h}$, the phosphorylation of Akt-mTOR-P70RSK and ERK-P90RSK-CREB signal pathways were determined by Western blot. Figures 4C and 5C showed that aloin could inhibit rhHMGB1induced the phosphorylation of the above signalling pathways.

\section{Inhibitory the activation of Akt $\mathrm{mTOR}$} and ERK enhanced Aloin induced HGC-

\section{7 cells apoptosis}

To prove Akt and ERK pathways involved in ALOinduced HGC-27 cell apoptosis, LY294002, Rapamycin 

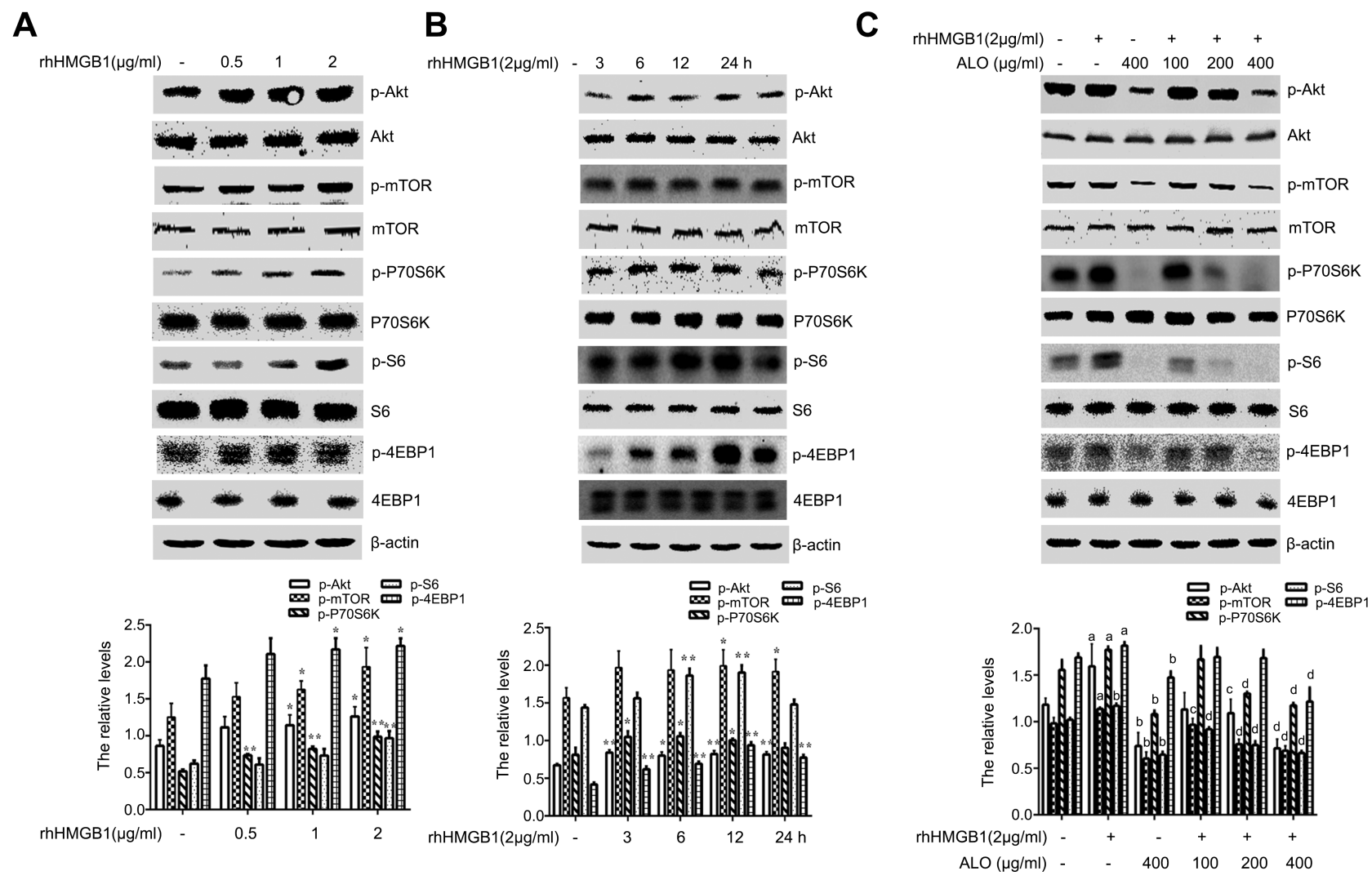

Figure 4 Aloin (ALO) inhibited the activation of the Akt-mTOR-P70S6K signalling pathway induced by rhHMGBI. HGC-27 cells were treated with different doses of rhHMGBI for $12 \mathrm{~h} \mathrm{(A)}$, or with $2 \mu \mathrm{g} / \mathrm{ml}$ of rhHMGBI at different times $(3,6,12,24 \mathrm{~h})(\mathbf{B})$, the phosphorylatio and total amount of Akt, mTOR, P70S6K, S6, 4EBPI were detected by Western blotting. The relative levels were normalized by $\boldsymbol{\beta}$-actin. Data are presented as mean \pm SD of three independent experiments. * $p<0.05$ and $* * *<0.01$ vs control group. HGC-27 cells were pre-treated with different doses of ALO for $2 \mathrm{~h}$, and then stimulated with $2 \mu \mathrm{g} / \mathrm{ml}$ of rhHMGBI for $12 \mathrm{~h}$ (C). The phosphorylation and total amount of Akt, mTOR, P70S6K, S6, 4EBPI were detected by Western blotting. The relative levels were normalized by $\beta$-actin. Data are presented as mean \pm SD of three independent experiments. ${ }^{a} p<0.05$ and ${ }^{b} p<0.01$ vs control group, ${ }^{c} p<0.05$ and ${ }^{d} p<0.01$ vs rhHMGBI group.

and U0126, the special inhibitor of Akt, mTOR and ERK were used. Firstly, the inhibitory effect of Rapamycin was investigated. HGC-27 cells were treated with different doses of Rapamycin $(25,50,100 \mathrm{nM})$ for 30 or $60 \mathrm{~min}$, the phosphorylation of mTOR was detected by Western blot. As shown in Figure 6A, Rapamycin suppressed phosphorylation of mTOR, In particular, $25 \mathrm{nM}$ of Rapamycin for $1 \mathrm{hr}$ exerted a significant inhibitory effect. Secondly, the inhibitory effect of LY294002, Rapamycin and U0126 on phosphorylation of Akt, mTOR and ERK respectively was tested by using Western blot. Figure 6B shows that treatment of HGC27 cells with LY294002 (15 $\mu \mathrm{M})$, Rapamycin (25 nM) and U0126 $(20 \mu \mathrm{M})$ decreased the phosphorylation of Akt, mTOR and ERK respectively. Finally, we examined ALO-induced inhibition of gastric cancer cell apoptosis. HGC-27 cells were pre-treated with inhibitor respectively for $1 \mathrm{~h}$, and then stimulated with ALO for $24 \mathrm{~h}$, DAPI staining and flow cytometry assay were used to detect gastric cancer cells apoptosis. Here, the inhibitor enhanced nuclear condensation and fragmentation induced byALO in HGC-27 cells (Figure 6C). The apoptotic rate in ALO treated cells was 22.31\%. However, LY294002, Rapamycin and U0126 pre-treatment all increased ALO-induced apoptosis to $36.31 \%, 32.99 \%$ and $30.32 \%$ respectively (Figure 6D).

In conclusion, ALO-induced apoptosis of gastric cancer HGC-27 cells by inhibiting the expression and release of HMGB1. ALO also suppressed HMGB1induced activation of Akt-mTOR-P70S6K and ERKP90RSK-CREB signalling pathways. These results provide new insight into the anti-tumor effects of ALO on gastric cancer cells.

\section{Discussion}

We investigated the molecular mechanisms of ALOinduced gastric cancer apoptosis, achieved by targeting HMGB1. ALO inhibited HGC-27 cell viability, and 
A

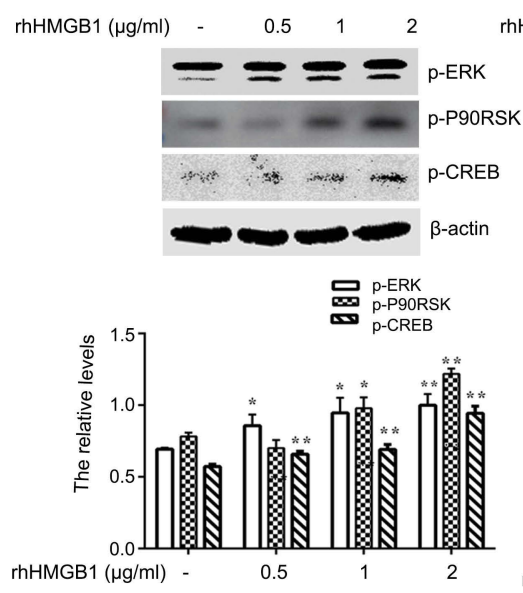

B
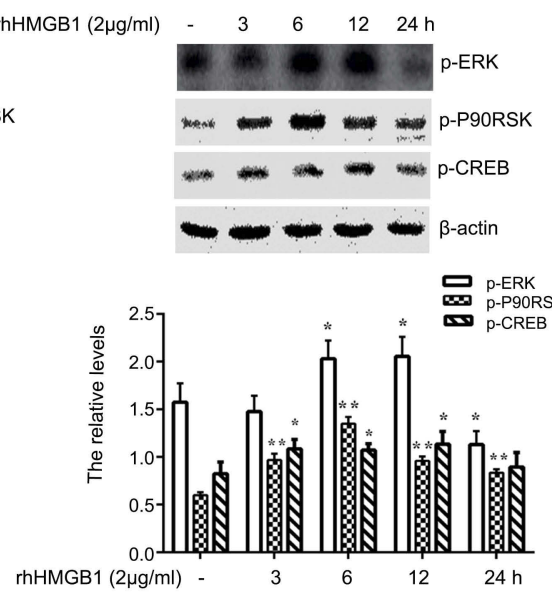

C
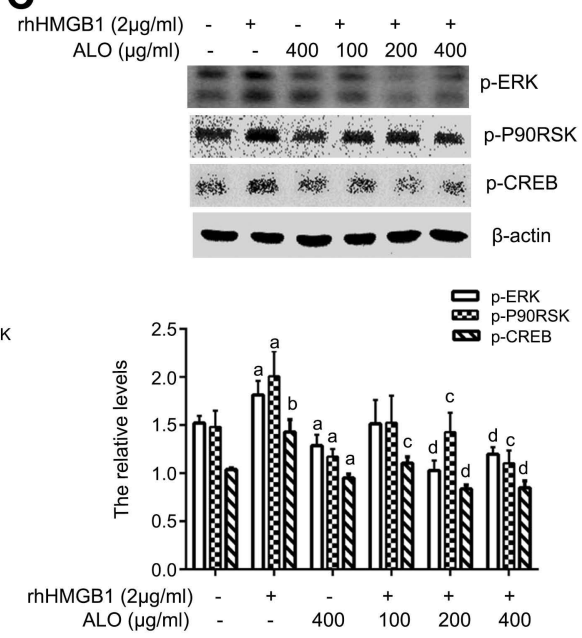

Figure 5 ALO inhibited the activation of the ERK-P90RSK-CREB signalling pathway induced by rhHMGBI in HGC-27 cells. HGC-27 cells were treated with different doses of rhHMGBI for $12 \mathrm{~h}(\mathbf{A})$, or with $2 \mu \mathrm{g} / \mathrm{ml}$ of rhHMGBI over different time points (B). The phosphorylation of ERK, P90RSK and CREB were detected by Western blotting. Data are presented as mean \pm SD of three independent experiments. ${ }^{*} p<0.05$ and ${ }^{* *} p<0.01$ vs control group. HGC-27 cells were pre-treated with different doses of $A L O$ for $2 \mathrm{~h}$, and then stimulated with $2 \mu \mathrm{g} / \mathrm{ml}$ of rhHMGBI for $12 \mathrm{~h}$ (C). The phosphorylation of ERK, P90RSK and CREB were detected by Western blotting. The relative levels were normalized by $\boldsymbol{\beta}$ actin. Data are presented as mean \pm SD of three independent experiments. ${ }^{a} p<0.05$ and ${ }^{b} p<0.01$ vs control group, ${ }^{C} p<0.05$ and ${ }^{d} p<0.01$ vs rhHMGBI group. Abbreviation: ALO, aloin.

induced their apoptosis (Figure 1). Although we have found that ALO could induce apoptosis of gastric cancer cells by regulating MAPKs signal pathway, ${ }^{17}$ the underlying mechanisms require further elucidation.

HMGB1 is highly expressed in cancer tissues and is closely related to the apoptosis, proliferation and migration of cancer cells. Anti-cancer therapies that target HMGB1 have attracted more and more attention. ${ }^{12,22}$ HMGB1 was more highly expressed in HGC-27 cells, compared to GES-1 cells (Figure 2A). These data suggest that HMGB1 may act as a biomarker for gastric cancer. Given this, we further explored the effects of ALO on HMGB1 expression. As shown in Figure 2B, ALO reduced HMGB1 expression in a concentration dependent manner. RAGE, a transmembrane protein, is also a receptor of HMGB1, and the expression of RAGE is closely associated with gastric and colorectal cancer invasion and metastasis of cancer. ${ }^{23,24}$ The HMGB1-RAGE signalling pathway plays an important role in the occurrence and development of tumor, ${ }^{25-27}$ and the accumulation of HMGB1 and RAGE activates a complex signalling network for cell survival and apoptosis evasion. ${ }^{28}$ Therefore, targeting of HMGB1-RAGE signalling could be a promising therapy for these types of cancer. As shown in Figure 2B, the expression level of RAGE also decreased after ALO treatment. Collectively, our results imply that ALO induces gastric cancer apoptosis by partially regulating the expressions of HMGB1 and RAGE partly.

Although at baseline HMGB1 was mainly localised to the nucleus, it can transfer to the cytoplasm and extracellular spaces. Released HMGB1 mediates diverse responses by binding to its receptors, then triggering various biological effects, including cell proliferation, differentiation and apoptosis. ${ }^{29}$ Therefore, we also explored the role of ALO in the release of HMGB1 in HGC-27 cells. As displayed in Figure 2C and D ALO clearly decreased the release and nuclear transposition of HMGB1. To further clarify the effect of HMGB1 on ALO-induced apoptosis, we down regulated HMGB1 expression by transfection of HMGB1 shRNA plasmids into HGC-27 cells. As shown in Figure 3, ALO clearly enhanced HGC-27 cell apoptosis in HMGB1 shRNA transfected cells compared with negative plasmid transfected cells. The above results guided us to explore whether ALO further activated the HMGB1-triggered intracellular signalling pathway, thereby inducing gastric cancer cell apoptosis.

The Akt-mTOR signalling pathway plays an important role in cell proliferation, survival, growth and apoptosis. ${ }^{30}$ The signalling pathway of the CREB is over-expressed and phosphorylated in many tumors, and is involved in cell proliferation, differentiation and survival. Many 
A

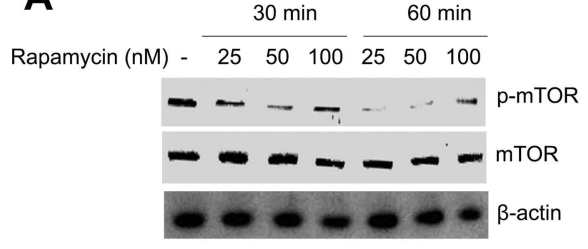

B

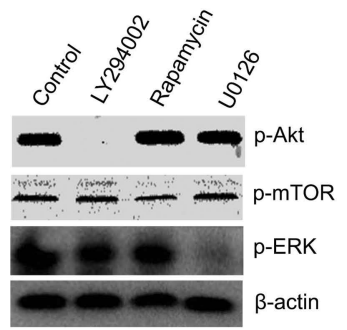

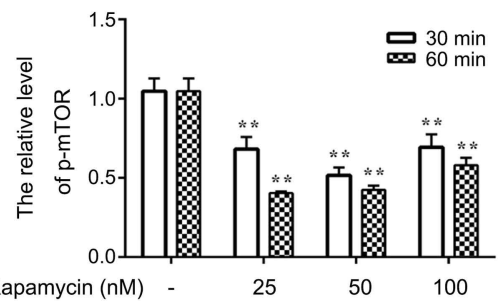

Rapamycin (nM)

100

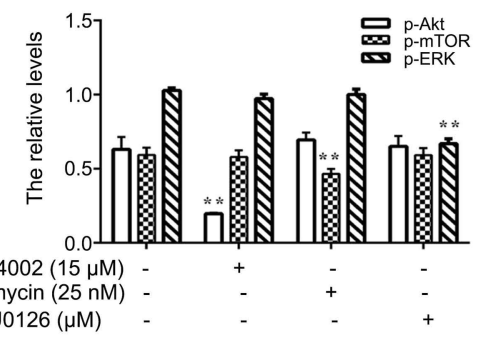

C
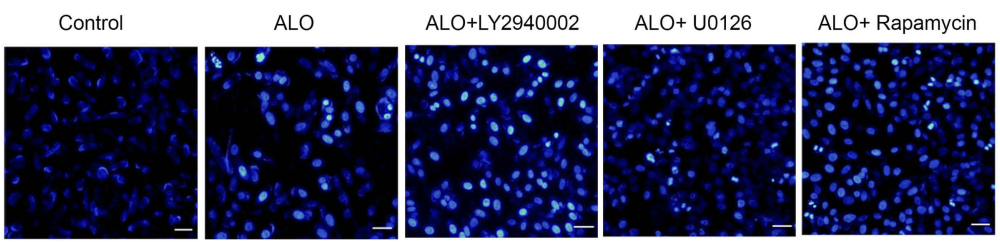

D
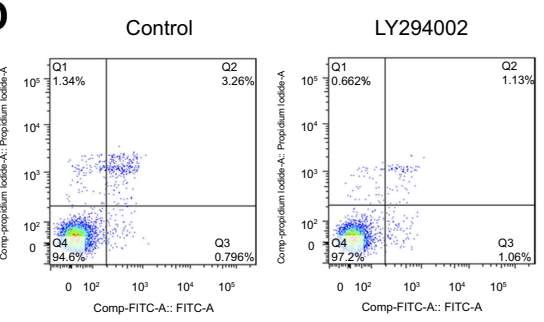

ALO+LY294002
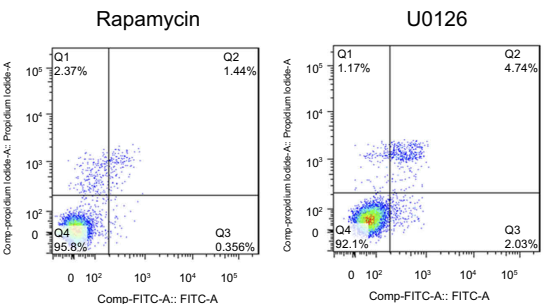

ALO
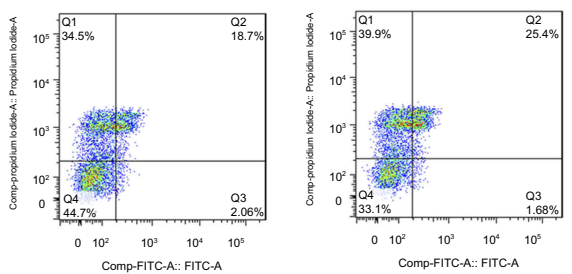

ALO+Rapamycin

ALO+U0126
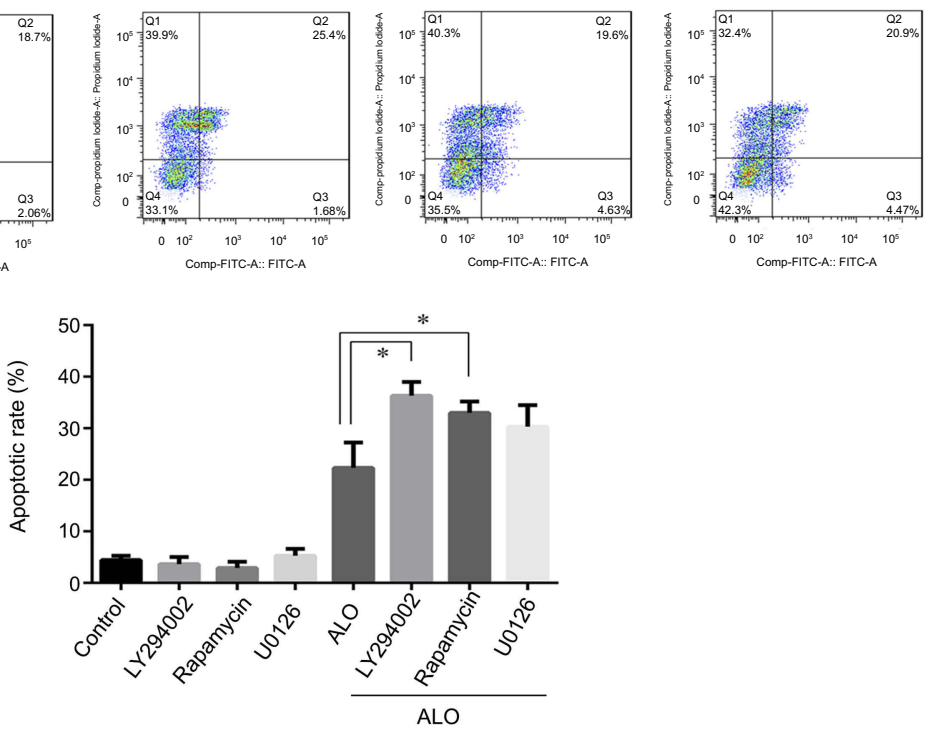

Figure 6 Inhibition of Akt, mTOR and ERK activation enhanced ALO-induced HGC-27 cells apoptosis. HGC-27 cells were treated with different doses of Rapamycin (25, 50 , I00 nM) for 30 or $60 \mathrm{~min}, \mathrm{p}$-mTOR and mTOR expressions were detected by Western blot (A). HGC-27 cells were treated with LY294002 (I5 $\mu$ M), Rampmycin ( $25 \mathrm{nM}$ ) or U0I 26 (20 $\mu$ M) for $\mathrm{I} \mathrm{h}$, the phosphorylation of Akt, mTOR and ERK was determined by Western blotting respectively (B). Data are presented as mean \pm SD of three independent experiments. ${ }^{*}<<0.05$ and $*_{p}^{*}<0.01$ vs control group. HGC-27 cells were pre-treated with LY294002 (I5 $\left.\mu \mathrm{M}\right)$, Rampmycin ( $\left.25 \mathrm{nM}\right)$ or U0I26 (20 $\left.\mu \mathrm{M}\right)$ for I h, and then $400 \mu \mathrm{g} / \mathrm{ml}$ of ALO and stimulated for another $24 \mathrm{~h}$. Nuclear morphology was observed by using DAPI staining (C). The apoptotic rate of HGC-27 cells was detected by flow cytometry (D). Abbreviation: ALO, aloin. 
kinases such as Akt, ERK, P90RSK, CaMK can induce phosphorylation of CREB. ${ }^{31}$ HMGB1-RAGE interaction regulates the proliferation, migration and apoptosis of cancer cells by activating a complex signalling network that includes Akt, ERK and NF-кB. ${ }^{8,20}$ Thus, inhibition of these signalling pathways could be potential target for many cancer therapies.

Our results showed that rhHMGB1 stimulated HGC-27 cells, enhanced the phosphorylation of the Akt-mTOR -P70S6K and ERK-P90RSK-CREB signalling pathways. ALO pre-treatment clearly inhibited rhHMGB1-induced these signalling pathways activation (Figures 4 and 5). In order to test Akt-mTOR and ERK pathways involved in ALO-induced HGC-27 cells apoptosis, we used their special inhibitors to block the activation of these signalling pathways. Blocking of Akt or mTOR activation obviously enhanced ALO-induced HGC-27 cell apoptosis. Inhibition of the ERK signalling pathway also enhanced gastric cancer cell apoptosis induced by ALO, but the effect was not significant (Figure 6). The results might suggest that HMGB1 triggers Akt-mTOR-P70S6K activation and may play a main role in ALO-induced HGC-27 cell apoptosis.

ALO can inhibit the nuclear translocation and release of HMGB1, but how does ALO affect the nuclear translocation of HMGB1? Post-translational modifications of HMGB1, such as phosphorylation, acetylation and methylation, are closely related to their nuclear translocation and release. ${ }^{10}$ Is ALOinduced inhibition of HMGB1 nuclear translocation related to its post-translational modification? How does ALO affect the expressions of HMGB1 and RAGE? Does other molecular mechanisms involved in ALO-induced HGC-27 cells death? These are the problems that require future exploration.

In conclusion, ALO promoted gastric cancer cell apoptosis by inhibiting the expression and release of HMGB1, and phosphorylation of ATK-mTOR-P70S6K and ERKP90RSK-CREB pathways. These results provide a new perspective for understanding the anti-tumor effect of ALO, as well as an experimental basis for HMGB1 as a potential target for the treatment of gastric cancer.

\section{Acknowledgments}

This work was supported by Natural Science Foundation of China (grant no. 81601380), Natural Science Research Project of Anhui Colleges and Universities (grant no. KJ2016SD59); Outstanding Young Talent Support Programme Key Projects of Anhui Colleges and Universities (grant no. gxyqZD2016173), Active Biological Macromolecules Research Provincial Key Laboratory Project (grant no.
1306C083008), National college Students‘ innovation and Entrepreneurship training programme project (grant nos. 201810368024 and 201710368002) and the Anhui Province College Students' Innovation and Entrepreneurship Training Programme project (grant no. 201710368166).

\section{Disclosure}

The authors report no conflicts of interest in this work.

\section{References}

1. Guo D, Zhang B, Liu S, et al. Xanthohumol induces apoptosis via caspase activation, regulation of $\mathrm{Bcl}-2$, and inhibition of PI3K/Akt/ mTOR-kinase in human gastric cancer cells. Biomed Pharmacother. 2018;106:1300-1306.

2. Suren D, Arda Gokay A, Sayiner A. High mobility group box 1 (HMGB1) expression in gastric adenocarcinomas. $J$ B.U.On.. 2018;23(2):422-427.

3. Tsung A, Klune JR, Zhang X, et al. HMGB1 release induced by liver ischemia involves Toll-like receptor 4 dependent reactive oxygenspecies production and calcium-mediated signaling. J Exp Med. 2007;204(12):2913-2923. doi:10.1084/jem.20070247

4. Wu X, Wang W, Chen Y, et al. High mobility group box protein 1 serves as a potential prognostic marker of lung cancer and promotes its invasion and metastasis by matrix metalloproteinase- 2 in a nuclear factor-kappaB-dependent manner. Biomed Res Int. 2018;2018:3453706.

5. Evankovich J, Cho SW, Zhang R, et al. High mobility group box 1 release from hepatocytes during ischemia and reperfusion injury is mediated by decreased histone deacetylase activity. J Biol Chem. 2010;285(51):39888-39897. doi:10.1074/jbc.M110.128348

6. Zeng W, Shan W, Gao L, et al. Inhibition of HMGB1 release via salvianolic acid B-mediated SIRT1 up-regulation protects rats against non-alcoholic fatty liver disease. Sci Rep. 2015;5:16013. doi:10.1038/ srep16013

7. Yang H, Tracey KJ. Targeting HMGB1 in inflammation. Biochim Biophys Acta. 2010;1799(1-2):149-156. doi:10.1016/j.bbagrm.2009.11.019

8. Song B, Song WG, Li ZJ, et al. Effect of HMGB1 silencing on cell proliferation, invasion and apoptosis of MGC-803 gastric cancer cells. Cell Biochem Funct. 2012;30(1):11-17. doi:10.1002/cbf.1811

9. Li Y, Tian J, Fu X, et al. Serum high mobility group box protein 1 as aclinical marker for ovarian cancer. Neoplasma. 2014;61(5):579-584. doi:10.4149/neo_2014_070

10. Richard SA, Jiang Y, Xiang LH, et al. Post-translational modifications of high mobility group box 1 and cancer. Am J Transl Res. 2017;9(12):5181-5196.

11. Lu L, Zhang D, Xu Y, Bai G, Lv Y, Liang J. miR-505 enhances doxorubicin-induced cytotoxicity in hepatocellular carcinoma through repressing the Akt pathway by directly targeting HMGB1. Biomed Pharmacother. 2018;104:613-621. doi:10.1016/j. biopha.2018.05.087

12. Zhang J, Kou YB, Zhu JS, Chen W-X, Li S. Knockdown of HMGB1 inhibits growth and invasion of gastric cancer cells through the NF-kappaB pathway in vitro and in vivo. Int $J$ Oncol. 2014;44 (4):1268-1276. doi:10.3892/ijo.2014.2285

13. Zhang QY, Wu LQ, Zhang T, Han Y-F, Lin X. Autophagy-mediated HMGB1 release promotes gastric cancer cell survival via RAGE activation of extracellular signal-regulated kinases 1/2. Oncol Rep. 2015;33(4):1630-1638. doi:10.3892/or.2015.3782

14. Wan L, Zhang L, Fan K, Wang J. Aloin promotes A549 cell apoptosis via the reactive oxygen speciesmitogen activated protein kinase signaling pathway and p53 phosphorylation. Mol Med Rep. 2017;16 (5):5759-5768. doi:10.3892/mmr.2017.7379 
15. Pan Q, Pan H, Lou H, Xu Y, Tian L. Inhibition of the angiogenesis and growth of Aloin in human colorectal cancer in vitro and in vivo. Cancer Cell Int. 2013;13(1):69. doi:10.1186/1475-2867-13-69

16. Esmat AY, Tomasetto C, Rio M-C. Cytotoxicity of a natural anthraquinone (Aloin) against human breast cancer cell lines with and without ErbB-2: topoisomerase IIalpha coamplification. Cancer Biol Ther. 2006;5(1):97-103. doi:10.4161/cbt.5.1.2347

17. Wang ZQ, Tao H, Ma YF, et al. Aloin induces apoptosis via regulating the activation of MAPKs signaling pathway in human gastric cancer cells in vitro. Nan Fang Yi Ke Da Xue Xue Bao. 2018;38 (9):1025-1031. doi:10.12122/j.issn.1673-4254.2018.09.01

18. He S, Cheng J, Feng X, Yu Y, Tian L, Huang Q. The dual role and therapeutic potential of high-mobility group box 1 in cancer Oncotarget. 2017;8(38):64534-64550. doi:10.18632/oncotarget.17885

19. Tang D, Kang R, Cheh CW, et al. HMGB1 release and redox regulates autophagy and apoptosis in cancer cells. Oncogene. 2010;29(38):5299-5310. doi:10.1038/onc.2010.261

20. Huang Z, Zhong Z, Zhang L, et al. Down-regulation of HMGB1 expression by shRNA constructs inhibits the bioactivity of urothelial carcinoma cell lines via the NF-kappaB pathway. Sci Rep. 2015;5:12807. doi:10.1038/srep12807

21. Zhao S, Jiang Y, Zhao J, et al. Quercetin-3-methyl ether inhibits esophageal carcinogenesis by targeting the AKT/mTOR/p70S6K and MAPK pathways. Mol Carcinog. 2018;57(11):1540-1552. doi:10.1002/ mc. 22876

22. Shen X, Hong L, Sun H, Shi M, Song Y. The expression of high-mobility group protein box 1 correlates with the progression of non-small cell lung cancer. Oncol Rep. 2009;22(3):535-539.

23. Kuniyasu H, Oue N, Wakikawa A, et al. Expression of receptors for advanced glycation end-products (RAGE) is closely associated with the invasive and metastatic activity of gastric cancer. J Pathol. 2002;196(2):163-170. doi:10.1002/path.1031
24. Sasahira T, Akama Y, Fujii K, Kuniyasu H. Expression of receptor for advanced glycation end products and HMGB1/amphoterin in colorectal adenomas. Virchows Arch. 2005;446(4):411-415. doi:10.1007/s00428005-1210-x

25. Fei Q, Xiao J, Gai L, et al. HMGB1-RAGE signaling facilitates Ras-dependent Yap1 expression to drive colorectal cancer stemness and development. Mol Carcinog. 2019;58(4): 500-510.

26. Wang L, Kang FB, Wang J, et al. Downregulation of miR-205 contributes to epithelial-mesenchymal transition and invasion in triple-negative breast cancer by targeting HMGB1-RAGE signaling pathway. Anticancer Drugs. 2019;30(3):225-232.

27. Li S, Gu H, Huang Y, et al. Circular RNA 101368/miR-200a axis modulates the migration of hepatocellular carcinoma through HMGB1/RAGE signaling. Cell Cycle. 2018;17(19-20):2349-2359. doi:10.1080/15384101.2018.1526599

28. Dhumale SS, Waghela BN, Pathak C. Quercetin protects necrotic insult and promotes apoptosis by attenuating the expression of RAGE and its ligand HMGB1 in human breast adenocarcinoma cells. IUBMB Life. 2015;67(5):361-373. doi:10.1002/iub.1379

29. Huang CY, Chiang SF, Chen WT, et al. HMGB1 promotes ERK-mediated mitochondrial Drp1 phosphorylation for chemoresistance through RAGE in colorectal cancer. Cell Death Dis. 2018;9 (10):1004. doi:10.1038/s41419-018-1019-6

30. Qin G, Li P, Xue Z. Triptolide induces protective autophagy and apoptosis in human cervical cancer cells by downregulating Akt/ mTOR activation. Oncol Lett. 2018;16(3):3929-3934. doi:10.3892/ ol.2018.9074

31. Sakamoto KM, Frank DA. CREB in the pathophysiology of cancer: implications for targeting transcription factors for cancer therapy. Clin Cancer Res. 2009;15(8):2583-2587. doi:10.1158/1078-0432. CCR-08-1137
Drug Design, Development and Therapy

\section{Publish your work in this journal}

Drug Design, Development and Therapy is an international, peerreviewed open-access journal that spans the spectrum of drug design and development through to clinical applications. Clinical outcomes, patient safety, and programs for the development and effective, safe, and sustained use of medicines are a feature of the journal, which has also

\section{Dovepress}

been accepted for indexing on PubMed Central. The manuscript management system is completely online and includes a very quick and fair peer-review system, which is all easy to use. Visit http://www. dovepress.com/testimonials.php to read real quotes from published authors. 